\title{
Hacia una explicación naturalizada del problema filosófico de las otras mentes
}

\author{
Anyerson Stiths Gómez Tabares \\ Universidad Católica Luis Amigó (Colombia) \\ José Fernando Ospina \\ Andrés Felipe Micolta Henao \\ Universidad de Caldas (Colombia)
}

Recibido: septiembre 13 de 2019. Revisado: octubre: 1 de 2019. Aceptado: diciembre 10 de 2019

Referencia norma APA: Gómez, A. S., Ospina, J. F., \& Micolta, A. F (2019). Hacia una explicación naturalizada del problema filosófico de las otras mentes. Rev. Guillermo de Ockham, 17(2), 61-70. doi: https://doi.org/10.21500/22563202.4269

\section{Resumen}

En este artículo se pretende mostrar que el tratamiento de algunos problemas tradicionales de la filosofía de mente — para el caso de este trabajo, el problema de las otras mentes - debe articularse a una visión naturalista del mundo, y en coherencia con ello, al estudio empírico de las ciencias cognitivas, de tal manera que la reflexión metateórica propia de la filosofía soporte sus principios metafísicos, epistemológicos y lógicos en el andamiaje de la evidencia empírica.

Se intentará utilizar una metodología naturalista basada en la revisión de teorías científicas con evidencia empírica en los campos de la neurociencia cognitiva, la etología y la psicología cognitiva sobre el problema filosófico de las otras mentes y de cómo el constructo científico de teoría de la mente (ToM) puede aportar a su solución o reformulación. Esto supone, ciertamente, una revaluación del problema filosófico, sus argumentos e intentos de solución, resultado de una naturalización fructífera anclada en una visión teleológica de la evolución cognitivo-social del ser humano y otras especies.

Palabras clave: teoría de la mente; otras mentes; epistemología naturalizada; ciencia cognitiva.

\section{Towards a naturalized explanation of the philosophical problem of the other minds}

\section{Abstract}

In this paper we will try to show that the treatment of some traditional problems of the philosophy of the mind, in the case of this work, the problem of the other minds, can be articulated with a naturalistic vision of the world, and in coherence with it, the empirical study of cognitive sciences, thus, the meta-theoretical reflection of the supporting philosophy of its metaphysical, epistemological and logical principles from the scaffolding of the empirical evidence.

We will try to use a naturalistic methodology based on the revision of scientific theories with empirical evidence in the field of cognitive neuroscience, ethology and cognitive psychology about the philosophical problem of other minds and how the scientific construct of the theory of mind (ToM) can provide a solution or reformulation. This means, undoubtedly, a re-evaluation of the philosophical problem, its arguments and attempts of solution. The result could be a fruitful naturalization and a teleological vision of the cognitive-social evolution of the human being and other species.

Keywords: Theory of the mind; other minds; naturalized epistemology; cognitive science. 


\section{Introducción}

En el campo de la filosofía de la mente se han cristalizado una serie de problemas relacionados con la explicación de la conciencia, la intencionalidad, las otras mentes y la causalidad, entre otros, desde posturas ontológicas, lógicas y epistemológicas, buscando de alguna manera descifrar las complejas relaciones entre categorías físicas y mentales y de cómo estas se arraigan en el funcionamiento del mundo físico. Dicha tarea no ha sido fácil. Desde la concepción cartesiana heredada del siglo XVII, se ha acentuado un dualismo casi irreconciliable entre lo físico y lo mental que ha llevado a los filósofos a defender todo tipo de ontologías para salvaguardar una explicación plausible de la conciencia. Se han defendido y debatido todo tipo posiciones, que van desde un dualismo de tipos o propiedades hasta ontologías monistas, sean de tipo idealista o materialista, lo que ha generado, en apariencia, más confusiones que soluciones.

El punto en cuestión que se pretende considerar como partida de este trabajo no reside en el tipo de explicación ontológica que se considere más loable por los filósofos ni los esfuerzos puestos en sus argumentos y demostraciones lógicas, sino la de cuestionar si realmente la filosofía está en condiciones para determinar qué es lo que existe o no en el mundo y robustecer o reducir la ontología de ciertas categorías que atañen a los problemas perennes de la filosofía de la mente, entre ellos, específicamente, la existencia de las otras mentes; o si por el contrario y en coherencia con la propuesta de Quine $(1960,1969,1975,1986)$ de naturalizar la epistemología volviéndola un capítulo de la ciencia empírica, concretamente de la psicología y las neurociencias, al ser las más capacitadas para determinar una explicación sobre la existencia de las otras mentes. En otras palabras, examinar las tesis filosóficas en coherencia con la evidencia y los métodos de la ciencia empírica.

Es de aclarar que la evolución de la teoría de Quine y su propuesta naturalista no debe, en últimas, ser considerada eliminativista, tal y como parece serlo en su obra Word and Object (1960). A pesar de que en este texto plantea la imposibilidad de una ciencia de la intencionalidad y de las expresiones mentales, años después clarifica posición su al considerar que no es posible eliminar el discurso intencional, lo cual lleva al autor a una visión moderada de lo mental en el sentido de que su epistemología se muestra más tolerante con la psicología popular desde el punto de vista de la descripción, lo cual no supone compromisos ontológicos (Quine, 1886, 1998). En este sentido, Quine se adhiere al monismo anómalo de Davidson (Quine, 1998, 2014).
Ahora bien, esta idea de tinte naturalista se sustenta en el hecho de que no hay un dominio de principios sobre una explicación de la naturaleza de lo mental que sea investigable aprioristicamente, y en este sentido ni la epistemología ni la filosofía de la mente tienen superioridad ni pueden tener por objeto un estudio de ese carácter. Por el contrario, la explicación filosófica de la existencia de las otras mentes debe considerarse en coherencia con una explicación científica de las otras mentes.

Por lo general, se ha considerado que la disciplina filosófica y el campo de las ciencias se han ocupado de problemas de distinta naturaleza, a veces incompatibles en cuanto a su nivel de reflexión teórica. De alguna manera, los filósofos han considerado que su disciplina se ocupa de problemas perennes que van más allá de la posibilidad de una solución con evidencia empírica y no sería de extrañar que justamente las preguntas formuladas por filósofos y científicos tuvieran ese carácter distintivo que evita establecer un puente entre ambos campos de conocimiento. Un buen ejemplo son los tipos de problemas que se formulan respecto a la explicación de lo mental. Por un lado, están aquellos problemas que se han cristalizado en la filosofía de la mente: la intencionalidad, la causación de lo mental, la fenomenología y la ontología de los rasgos mentales, la relación mente-cuerpo, y más taxativamente, en lo que concierne este trabajo, la explicación de las otras mentes. Gran parte del soporte explicativo de estos problemas reside en el campo del análisis del lenguaje mental, la ontología, la lógica y la epistemología.

De otro lado, la ciencia, específicamente la neurociencia cognitiva, la etología cognitiva, la psicología del desarrollo de corte cognitivo, la neuropsicología y la psicología cognitiva, se han ocupado de lo mental desde un punto de vista funcional del cerebro y su relación con la conducta social y el funcionamiento psicológico, de tal suerte que sus hallazgos han permitido establecer evidencia empírica considerable para explicar lo mental en términos del funcionamiento cerebral ligado a procesos socioculturales. Lo interesante de la cuestión es que la preocupación de las ciencias recae en la evidencia empírica y no en el análisis de las implicaciones ontológicas de sus hallazgos, a pesar de poseer ciertos soportes empíricos a su favor. Sin embargo, estos compromisos ontológicos son reflexionados en el campo de la filosofía y no parecen ser una preocupación propiamente científica. La naturaleza misma de las categorías mentales y su relación con lo cerebral se establece en términos empíricos y no lógicos u ontológicos, y justamente las relaciones lógicas entre categorías mentales y cerebrales y la ontología de la conciencia son asuntos que han ocupado, generalmente, a los filósofos. 
En este sentido, llama la atención cómo dos tipos de saberes con niveles diferentes de reflexión, a saber, las ciencias cognitivas y la filosofía de la mente, se han ocupado de problemas que requieren una articulación y un diálogo interdisciplinar con lo ontológico, lo epistémico y lo empírico, que apenas recientemente se está explorando. Con este panorama se ha generado la impresión de que los problemas de la filosofía de la mente son inabordables para el campo de las ciencias cognitivas y que la evidencia de las neurociencias no podría solucionar o reformular problemas filosóficos. Sin embargo, esto no es así; por el contrario, parece que las fronteras entre la filosofía de la mente y las neurociencias cognitivas se han ido derrumbando a la luz de la evidencia empírica sobre el funcionamiento del cerebro y los correlatos con categorías mentales o psicológicas. Estos diálogos interdisciplinares entre la psicología, las neurociencias y la filosofía de la mente han permitido reflexionar sobre muchos de los problemas importantes sobre la explicación de lo mental, lo cual implica un diálogo disciplinar, plural y abierto que aporta al entendimiento de lo mental.

Muchos filósofos, entre ellos Searle (2006), Dennett (1988), Quine $(1969,1975)$ y Churchland $(1988,1995)$, entre otros, han considerado la importancia de naturalizar el conocimiento filosófico en lo que respecta la conciencia, y que muchos problemas de la filosofía, en este caso de la mente, realmente deben ser considerados a la luz de la evidencia científica. Si bien muchos de estos pensadores tienen ideas y definiciones distintas de lo que significa naturalizar la filosofía, lo que parecen compartir es que algunos problemas filosóficos respecto a lo mental deben ser considerados a la luz de la psicología y las neurociencias. No sería de extrañar que una postura de tal tipo sea atractiva tanto para filósofos como para científicos cognitivos y que de alguna manera sea un puente para la reflexión interdisciplinar. Autores como Searle y su defensa de la tesis del naturalismo biológico para explicar el nexo causal entre el cerebro y la mente; Quine $(1969,1975)$ y su postura de naturalizar la epistemología a la psicología científica; ${ }^{1}$ la postura de Churchland $(1988,1995)$ de un naturalismo científico y hasta cierto punto considerado eliminativista (Churchland, 1995) ${ }^{2}$ o Bennett (2003,
2008) y su incursión filosófica al campo de las neurociencias cognitivas, son algunos de muchos referentes de este puente interdisciplinar que se está tendiendo en las últimas décadas. En el caso de las neurociencias pasa algo similar. Autores como Damasio (2011), Llinás (2003), Iacoboni (2009), Bennett (2008) y Tonnoni (Tononi \& Koch, 2008), entre otros, han buscado establecer explicaciones empíricas a problemas comenzados en la filosofía y que de alguna manera aportan a la solución o disolución del problema.

De acuerdo con esto, se intentará utilizar una metodología naturalista basada en la revisión de teorías científicas con evidencia empírica en el campo de la neurociencia cognitiva, la etología y la psicología cognitiva, sobre el problema filosófico de las otras mentes y de cómo el constructo científico de la teoría de la mente (ToM) puede aportar a su solución o reformulación. Esto supone, ciertamente, una revaluación del problema filosófico, sus argumentos e intentos de solución, resultado de una naturalización fructífera anclada en una visión teleológica de la evolución cognitivo-social del ser humano y otras especies.

\section{Hacia una idea general de naturalización}

El naturalismo y los proyectos de naturalización de la epistemología asumen muchas posturas, interpretaciones y formas de establecerse, tanto en su definición como en las propuestas filosóficas defendidas (Hacker, 2006). La variedad de tesis naturalistas hace que el término y su definición sean dependientes de la postura filosófica adoptada. Sin embargo, no es el caso hacer una taxonomía de los diferentes tipos de naturalismo filosófico y los argumentos que los sustentan, pues esta tarea constituye por sí sola otra investigación filosófica con muchas aristas por explorar. Lo que se quiere dejar claro es que el proyecto de naturalizar la epistemología al campo de las ciencias empíricas ha sido prometedor para construir reflexiones conjuntas respecto de lo mental, que de cierta manera permite la discusión pluralista y conjunta en términos ontológicos, epistemológicos, metodológicos en relación con la evidencia empírica lograda. De otro lado, es importante precisar,

1. Si bien Quine en su texto sobre epistemología naturalizada (1969 [1984]) sugiere que la epistemología debe ser considerada como un capítulo de la psicología, en su artículo Mind and verbal dispositions (1975), sugiere que las neurociencias, específicamente, la neurofisiología es uno de los campos científicos sobre los cuales se podría naturalizar una explicación de las categorías mentales. Quine (1975) hace una distinción de tres niveles explicativos: 1. el nivel mental, que para el autor es superficial y no debe entenderse en términos ontológicos; 2 . el nivel fisiológico, el cual considera la base de la explicación causal de lo mental, aquí estaría la idea de una naturalización de la filosofía a la neurociencia y, finalmente; 3. el nivel conductista que es preferible al lenguaje mentalista. En este nivel estaría la psicología conductista y debe ser usado mientras no tengamos una explicación adecuada del funcionamiento de los procesos neurofisiológicos que serían la verdadera referencia del lenguaje mentalista. Desde los años setenta, Quine mostró su simpatía por la teoría de la identidad psicofísica, aunque la criticó por ser demasiado permisiva con el uso del lenguaje mentalista.

2. Es considerado eliminativista en el sentido de que defiende la idea de que la conciencia es reductible, estrictamente, a procesos de la función cerebral, y que términos propios de la psicología popular, como la intencionalidad, las creencias, los deseos y la subjetividad, no tienen un sustrato neurológico definido, y, por tanto, deben ser eliminadas del discurso científico y en algún momento del lenguaje en general. 
con mayor detalle, la idea de naturalismo que se quiere a defender y aceptar, con el fin de evitar malentendidos y discusiones innecesarias.

En el dominio del análisis ontológico y epistemológico de las categorías mentales, un naturalismo puede entenderse de la siguiente manera:

1. Toda teoría filosófica de la mente debe aceptar solo la existencia de entidades, estados, procesos o fenómenos de tipo material o físico, los cuales se pueden ubicar en términos espaciales, temporales, computacionales, funcionales o dinámicos, evitando de este modo, incluir categorías sustancialistas o entidades abstractas que no tengan un correlato con una visión física del mundo. De acuerdo con Clark (2016), Scotto (2017) y Prades (2006), esta visión implica asumir un principio metafísico de uniformidad y homogeneidad de la naturaleza de lo mental, y es que, justamente, debe funcionar bajo principios materiales.

2. Si toda explicación de los fenómenos mentales y de manera más concreta, la existencia de las otras mentes, la intencionalidad, la conciencia, entre otros, debe considerarse bajo una concepción natural del mundo (naturalismo ontológico), y, por tanto, materialista, pues se debe, por consiguiente, buscar una explicación de estos fenómenos en las ciencias, sea la biología, la etología, la psicología cognitivo-social, o las neurociencias y no en la mera especulación filosófica. Este supuesto implica no solo un naturalismo ontológico sino también epistemológico, en el sentido de que son los métodos y las técnicas de las ciencias empíricas los que pueden generar conocimiento sobre el funcionamiento de los fenómenos mentales en relación a sus correlatos moleculares, neurales, cerebrales y conductuales. Este tipo de naturalismo es coherente con el proyecto naturalista de Quine (1998), al considerar que la epistemología y los problemas allí planteados deben ser considerados a la luz de una comprensión científica del mundo. En otras palabras, los problemas acuciantes a la explicación de lo mental deben ser abordados bajo un tratamiento científico, sea conductista, computacional o neurocientífico, sin que esto implique eliminar el lenguaje intencional o la psicología folk. Es de mencionar que la visión naturalista de Quine se fue flexibilizando con los años, evidenciando con ello en la obra del estímulo a la ciencia, una postura moderada cercana, como ya se indicó, al monismo anómalo.

Ahora bien, ¿qué tipo de materialismo se está planteando? En la literatura filosófica hay discrepancias en cuanto a la forma de ser naturalista o de concebir la naturalización (Acero, 2012; Hacker, 2006). Por un lado, está el naturalismo considerado eliminativista, y por el otro, un naturalismo débil que no implica reducción o eliminación.

Un proyecto de naturalización reductiva plantea que las propiedades, estados, eventos o procesos mentales y todo lo que representa una descripción de estas categorías en todos los niveles explicativos, debe ser reductible a las propiedades materiales del cerebro. Esta distinción de conceptos es relativa al uso de cada autor. Por ejemplo, Davidson (1994) y hasta cierto punto Quine (1998), hacen referencia a eventos. Desde el punto de vista del funcionalismo computacional de Churchland (1995), se habla de procesos y no de eventos o estados. Ahora bien, más allá de las implicaciones epistémicas y ontológicas de estas categorías y las diferencias de grano a la hora de referirse a lo mental —lo cual constituiría un trabajo teórico más allá del aquí expuesto - lo que se quiere plantear es que una postura reductiva exige que la relación causal de las categorías mentales, sea cual fuera, debe estar determinada por una explicación material, sea molecular, neural, cerebral, fisiológica, o conductual. Este tipo de materialismo tan estricto como el propuesto por Churchland $(1988,1995)$ y Stich (1983), se rechaza justamente porque elimina la psicología popular (Dennett, 1988b) y la posibilidad de hacer una explicación psicológico-científica de lo mental.

El otro tipo de naturalismo, el cual se considera el más viable para los fines de este trabajo, es aquel que no implica eliminación de la psicología folk o del lenguaje con contenido psicológico. Para esto, se hace uso de un naturalismo instrumental (Fodor, 1985; Dennet, 1988b), en el que plantea que no es posible eliminar la psicología popular como otro nivel explicativo y descriptivo, y de este modo, aunque no existen elementos de hecho para ubicar las categorías mentales con una ontología particular o diferente al estado de cosas materiales, tampoco es posible eliminar el lenguaje psicológico en la explicación de los procesos mentales; es pragmáticamente inviable. Bajo esta perspectiva, el tipo de naturalismo adoptado es más bien abierto, pluralista e instrumental, en el sentido de que se propone establecer unos puentes de diálogo y conexión entre lo que Dennett denomió la estrategia intencional, la estrategia física y la estrategia del diseño. Dicho de otro modo, una relación entre la explicación especulativa de las otras mentes y las bases biológicas, cognitivas y sociales que lo sustentan.

Con lo expuesto anteriormente, es claro que el tipo de naturalismo propuesto aquí para una explicación científica de las otras mentes es no eliminativo, y que, en

$64<$ Universidad de San Buenaventura, Cali, Colombia 
coherencia con la propuesta de Quine (1988) de buscar en la psicología científica una fuente de fundamentación de las explicaciones filosóficas, en este caso la existencia de las otras mentes, es el marco metateórico de este trabajo y el concepto de teoría de la mente (ToM) es el constructo que busca reorientar la explicación de las otras mentes.

\section{El problema filosófico de las otras mentes}

Una manera de formular el problema filosófico seria de la siguiente manera: ¿cómo sé que otras personas tienen mente? O ¿cómo sé que hay mentes además de la mía? De manera directa solo es posible dar cuenta de la existencia de la mente en la perspectiva de la primera persona; sin embargo, ¿cómo puedo dar cuenta de la existencia de otras mentes cuando la única evidencia directa es la mente propia? Este problema versa sobre la tesis del lenguaje privado y la inaccesibilidad del contenido mental en los demás de manera directa.

Desde la segunda meditación, Descartes (2005 [1647]) sostiene la idea de que los contenidos del pensamiento solo pueden ser accesibles desde el punto de vista de quien los tiene, y que solo es posible tener certeza del yo (cogito), un contenido de pensamiento accesible solo mediante la introspección. Lo demás, los contenidos mentales de otras personas, la existencia de otros seres pensantes, el cuerpo y los objetos físicos pueden ser objeto de la duda hiperbólica y por consiguiente, no hay certeza, en este caso, de la existencia de otras mentes o de la accesibilidad para el contenido del pensamiento desde la tercera persona.

De esta manera, la única certeza epistémica es el cogito. Esta posición puede considerarse introspeccionista, antropocéntrica, egocéntrica e híperintelectualista. La tesis del lenguaje privado implícita en esta filosofía, parte del supuesto de que el lenguaje mentalista está compuesto por conceptos o palabras que describen sensaciones, emociones o pensamientos que solo pueden ser conocidos por el hablante y que en ningún caso otra persona puede tener acceso a los objetos a los que se refiere dicho lenguaje.

Esta tesis fue criticada fuertemente por Wittgenstein $(1953,1996)$, quien establece que todo lenguaje independiente de si hace o no referencia a sensaciones, creencias o deseos, debe estar caracterizado por criterios de uso públicos y sociales — si se quiere-, pues de lo contrario sería imposible establecer reglas que hicieran posible distinguir los usos lingüísticos correctos de los uso incorrectos.
Ahora bien, esta forma radical de plantear el problema epistemológico de las otras mentes y su anclaje en la tesis de un lenguaje privado, ubica lo mental como un conjunto de actos de pensamiento privado, solitario e individual

La aceptación de esta premisa lleva a la imposibilidad del acceso cognitivo de la mente en la perspectiva de la tercera persona, lo cual ha generado múltiples hipótesis y conclusiones extravagantes y no es de extrañar que el problema en los términos planteados se convirtiera en un reto importante en la explicación teórica, dado la accesibilidad directa a los estados mentales internos, en cambio, en la perspectiva de la tercera persona no es posible disponer de tal certeza cognitiva o acceso directo, lo cual genera una asimetría respecto al acceso de lo mental a favor de la primera persona.

De este modo, la forma de acceso a las otras mentes seria de manera indirecta o inferencial, en este caso, se requiere de un tipo de razonamiento o inferencia conceptual a partir del registro conductual o fisiológico de la otra persona para justificar la creencia de que los demás también tienen mente o estados mentales, pero ¿cómo establecer este nexo causal entre los estados mentales propios y la conducta de los demás, para justificar la existencia de las otras mentes?

Hay muchos argumentos y tesis que apelan a la imposibilidad causal, incluso inferencial, de dar cuenta de la existencia de las otras mentes; hay posiciones que apelan a argumentos trascendentales, teístas, incluso místicos, que no se considerarán aquí. Nagel (2003), por otro lado, plantea una tesis fenoménica del contenido de lo mental que estaría a favor de la imposibilidad del acceso cognitivo desde el punto de vista de la tercera persona, dado que, los deseos, sensaciones, creencias, y todo tipo de experiencias subjetivas solo la persona que los experimenta puede conocer y dar cuento de estos. De esta manera, la fenomenología de la experiencia de Nagel, plantea la imposibilidad de acceso de la experiencia fenoménica de los demás. Dicho de otra manera no es posible saber que se siente ser un murciélago (Nagel, 2003).

Ahora bien, ¿cómo poder dar una respuesta positiva al problema desde el punto de vista filosófico y poder justificar la existencia de las otras mentes? una salida que han utilizado los filósofos para esta cuestión es mediante el argumento por analogía, en el que a partir de la semejanza instituida en las premisas entre dos o más objetos en uno o varios aspectos, se colige la similitud de otro $\mathrm{u}$ otros objetos. 


\section{El argumento por analogía}

Dado que solo se tiene acceso directo a una sola mente (la propia), la única forma de dar cuenta de que los demás tienen también una mente es a través de su comportamiento. De acuerdo con diversos autores (Brunsteins, 2011; Searle, 2006; Scotto, 2017), los registros comportamentales son la clave del argumento por analogía para entender lo que sucede en las otras mentes. Para comprender cómo funciona esto es necesario un ejercicio analógico del siguiente tipo: si analizo mis propios estados mentales y su actividad en relación con mi cuerpo, ${ }^{3}$ me puedo dar cuenta de que hay un enlace entre mi mente y mi cuerpo. Por ejemplo, si estoy nervioso es posible que mi cuerpo sude así no esté haciendo calor; si tengo dolor, es posible que grite y así pueden darse muchos casos de la relación entre estados mentales y la expresión conductual o fisiológica. De este modo, un sujeto puede percibir a otra persona y encontrar una analogía entre su cuerpo y el propio y establecer, por consiguiente, una analogía entre las respuestas del cuerpo de la otra persona y la mente propia. De esta manera, al ver una persona sudando cuando no está haciendo calor, puedo inferir que está nerviosa; si la veo gritando, puedo inferir que tiene un estado mental de sufrimiento o dolor. En palabras de Searle (2006): "(...) cabría estimar que puedo inferir la existencia de estados mentales en ti por analogía conmigo mismo" (p. 33). En párrafos siguientes amplía la explicación del argumento: "[...] así como observo una correlación de mi comportamiento con mis estados mentales, puedo inferir la presencia de estados mentales correspondientes en otros al observar su comportamiento" (p. 51). Una manera de esquematizar el argumento sería el siguiente (Figura 1):

Figura 1

Esquema general del argumento por analogía

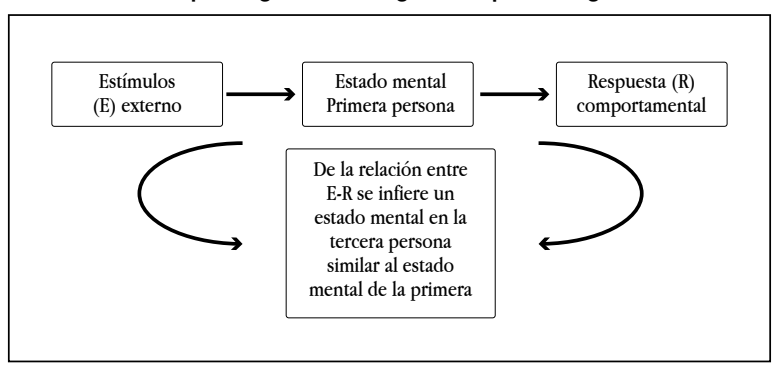

Fuente: construcción propia

De acuerdo con la Figura 1, el sujeto de la primera persona establece una relación entre un estímulo externo, su estado mental interno $x$, y una expresión conductual o respuesta fisiológica a dicho estado, de tal manera que en el caso de otras personas, al advertir un estímulo y una res- puesta o conducta determinadas se infiere por analogía un estado mental similar al del sujeto de la primera persona.

En razón de lo anterior, el argumento por analogía no parece resolver el problema. Por el contrario, parece que reafirma una visión de inaccesibilidad directa de lo mental en la perspectiva de la tercera persona. Esta crítica se fundamenta en dos sentidos. El primero, es considerar que la explicación sobre la existencia de las otras mentes se reduce a patrones de respuesta fisiológica o conductual de estímulos ambientes, que en ultimas puede no dar cuenta de una inferencia correcta de las otras mentes, pues el acceso directo, como ya se indicó, solo es posible en la primera persona, y lo segundo, de considerar que el registro fisiológico o conductual es el único elemento necesario para dar cuenta de las otras mentes, tal hipótesis tendría que ser objeto de verificación científica y no filosófica, es decir, no habría un problema filosófico de las otras mentes desligado a un problema científico de estas, dado que, si la conducta y el análisis de la misma es un elemento necesario para los procesos de razonamiento inferencial de las otras mentes, tal hipótesis debe encontrar su respuesta en la psicología cognitiva conductual, y, por tanto, en la verificación empírica. De ser así, el argumento por analogía, más que un campo de estudio filosófico, es una hipótesis científica que debe ser demostrada mediante la experimentación y no solo mediante el análisis lógico.

Otro punto problemático es que este argumento es otra forma de conductismo psicológico en el sentido en que son los procesos asociativos entre el estímulo (input) y la respuesta (output) los que determinan la inferencia de un estado mental en la tercera persona. En el desarrollo actual de la filosofía de la mente, la psicología y la neurociencia cognitiva sería un error recurrir al conductismo lógico y psicológico como la principal o única herramienta para explicar la existencia de las otras mentes.

Finalmente, una de las críticas más comunes apunta a que un razonamiento de este tipo para explicar las otras mentes es demasiado complejo para algo que parece darse de una manera espontánea y natural en la vida cotidiana. Sin grandes esfuerzos y con suma rapidez, una persona lee la mente de los demás independientemente de que tal lectura resulte acertada.

En vista de este panorama y de la dificultad de explicar la capacidad de dar cuenta de las otras mentes mediante argumentos meramente filosóficos e hipótesis conceptuales, los filósofos de la mente se han unido a neurocientíficos y psicólogos cognitivos en un intento de comprender

3. En este caso, la expresión mi propio cuerpo debe entenderse en términos de actividad conductual y fisiológica.

$66<$ Universidad de San Buenaventura, Cali, Colombia 
cómo es posible leer la mente de otra persona y de cómo la investigación científica en el campo de la teoría de la mente ha permitido una mayor comprensión del problema y ha dotado de evidencia empírica las visiones teóricas dominantes en el campo de la neurociencia cognitiva: la teoría-teoría y la teoría de la simulación.

\section{Estudios en teoría de la mente. Una mirada naturalista al problema filosófico}

La ciencia cognitiva contemporánea, mediante el desarrollo de técnicas avanzadas de neuroimagen y el desarrollo de metodologías experimentales en psicología cognitiva, ha logrado establecer las bases materiales (neurales, cerebrales y conductuales) asociadas a diversos procesos cognitivos (mentales) y sociales (conducta social), que de alguna manera han aportado significativamente a la comprensión de diversos fenómenos mentales discutidos por la filosofía y la psicología en sus albores. Uno de estos problemas es el de la existencia de las otras mentes. Desde un punto de vista empírico y epistemológico, la ciencia de orientación cognitiva ${ }^{4}$ se ha preguntado sobre la capacidad de los animales humanos y no humanos para inferir estados mentales en los demás y cuáles son los mecanismos funcionales del cerebro que lo explican, así como la función del medio en términos sociales, culturales $o$ institucionales que influyen en el tipo de inferencia que se hace del estado mental en la tercera persona.

En los años setenta, Premack y Woodruff, (1978) comenzaron en el campo de la etología cognitiva y comparada, uno de los principales trabajos empíricos sobre la capacidad de los chimpancés para predecir acciones humanas básicas y cómo la conducta de este primate daba cuenta de su capacidad para atribuir al humano estados mentales. Mediante la evidencia empírica se determinó que los chimpancés disponían de una teoría de la mente (ToM) en el sentido de un sistema inferencial que le permite predecir la conducta de otros chimpancés $\mathrm{y}$ animales humanos.

Es importante considerar que los investigadores no estaban buscando postular la existencia de una teoría de la mente en chimpancés en el mismo sentido que lo podría ser para un animal humano, sino plantear, mediante la evidencia empírica, que existe un sistema inferencial de carácter material (biológico-cerebral) que le permite al animal no humano predecir la conducta de otros chimpancés. Así lo plantean más adelante en su trabajo: "Con toda probabilidad las pruebas aquí planteadas distan mucho de aprehender completamente el nivel de abstracción del concepto de problema que tienen estos animales" (Premack y Wodruff, 1978, p. 151).

Lo más representativo del estudio de Premack y Woodruff, (1978) fue plantear la posibilidad de atribuir facultades representacionales y disposiciones inferenciales en el sentido de una teoría de la mente en animales no humanos cercanos filogenéticamente al animal humano, mediante elementos empíricos y metodologías experimentales en etología comparada, estableciendo de este modo un cambio paradigmático en la manera de entender el problema filosófico de las otras mentes, salir de las posturas antropocéntricas dominantes sobre la cognición y establecer una base empírica a la pregunta ¿cómo sé que otras personas tienen mente?

A pesar de las críticas iniciales al trabajo de Premack, \& Wordruff (1978), la utilización del constructo de teoría de la mente (ToM) en la investigación empírica se amplió a otros dominios investigativos. Desde la década de los ochenta, en el campo de la psicología del desarrollo de orientación cognitiva, diversos investigadores (Perner, \& Wimmer, 1985, 1987; Wimmer, \& Perner, 1983; Leslie, \& Frith, 1988; Leslie, 1992; Wellman, 2014; Wellman, \& Liu, 2014) iniciaron el estudio del constructo de teoría de la mente en niños y la capacidad que tenían para inferir comportamientos, creencias y deseos en los demás. En últimas, investigar la intencionalidad en el desarrollo cognitivo en infantes. Con base en los criterios normativos sugeridos por Dennett, se diseńaron metodologías experimentales que permitían dar cuenta de la capacidad de los niños para inferir estados mentales en la perspectiva de la tercera persona.

En uno de los primeros trabajos psicológicos llevados a cabo por Wimmer, \& Perner (1983), utilizaron tareas de corte experimental conocida como la tarea de la falsa creencia, dado que, para los autores " (...) la comprensión de las falsas creencias en otras personas requiere una representación explícita del error de la creencia de la persona en relación con su propio conocimiento" (Wimmer, \& Perner, 1983, p. 103). De este modo, la comprensión de la creencia falsa requiere procesos cognitivos complejos para diferenciar entre el estado de cosas del mundo y las creencias, deseos e intenciones en la primera y tercera personas como criterio para predecir las acciones de los demás. En últimas, la capacidad del niño para hacer inferencias y predicciones del estado mental en la perspectiva de tercera persona.

4. Neurociencia cognitiva, neuropsicología, psicología cognitiva, etología cognitiva, antropología evolucionista. 
Otro campo de investigación científica es el planteado por Baron-Cohen, Leslie, \& Frith (1985), quienes estudiaron el constructo de teoría de la mente en el espectro autista y confirmaron la hipótesis de que las personas con autismo no disponían de una ToM en el sentido de una capacidad para atribuir y diferenciar estados mentales en la primera y la tercera personas.

De acuerdo con Benavides-Delgado, \& RoncancioMoreno (2009), desde los años noventa hasta la actualidad se han efectuado cambios en cuanto a la revisión de los diseños, metodologías y tareas experimentales de las investigaciones en teoría de la mente como campo de estudio interdisciplinar en ciencias cognitivas y se ha mostrado un marcado interés en la predicción y los marcadores biopsicosociales de la ToM desde un punto de vista evolutivo. En la actualidad, se han consolidado áreas de investigación de orden neural que explican el correlato biológico de la ToM y la injerencia de los estímulos ambientales en términos sociales para la activación funcional de cierto tipo de neuronas catalogadas como neuronas en espejo (Iacoboni, \& Dapretto, 2006; Iacoboni, 2009; Pineda, Moore, Elfenbeinand,\& Cox, 2009), con el fin de lograr una mayor comprensión de los marcadores biológicos relacionados con la capacidad de una persona para atribuir creencias, deseos y otros estados mentales en los demás.

En el caso de las neurociencias y su explicación de la ToM se ha logrado establecer modelos de explicación empírica sobre la capacidad de inferir estados mentales en los demás. Dicho de otra manera, explicar empíricamente cómo una persona puede dar cuenta de la existencia de las otras mentes. Muchos de los estudios científicos en neuronas en espejo, la evidencia científica en etología y neurociencia cognitiva de la ToM muestran justamente una visión funcionalista materialista.

Es importante aclarar que la alusión a un funcionalismo es en el sentido de que un animal humano al cual se le atribuya vida mental, debe poseer un conjunto de procesos funcionales dinámicos instanciados físicamente (cerebro), independientemente del tipo de descripción, sea esta molecular, neural, cerebral, fisiológica o conductual. Desde este panorama, se diría que en el ser humano y en algunas especies no humanas, si tiene teoría de la mente es porque tiene neuronas en espejo. Ahora bien, considerar que el papel funcional de la ToM lo cumple los procesos funcionales en el campo neural, supone cierta afinidad con el funcionalismo y más específicamente con las teorías de especificación funcionales (Functional Specification Theories- FSTS) propuestas por Lewis (1983), en cuanto un estado mental, en este caso una inferencia o predicción de creencias en la perspectiva de la tercera persona, es un realizador particular del papel funcional del sistema de neuronas espejo, de tal modo que la ToM es cualquier proceso dinámico y funcional cerebral y de conexiones neurales que desempeñe la función de creencia apropiada de inferencia o predicción, con base en el entorno físico y sociocultural.

Las teorías dominantes en este contexto son la teoría -teoría (Wellman, 1990, 2014), la teoría de la simulación (Goldman, 2006, 2009) con su variante de la simulación corporeizada o teoría enactiva (Ezequiel, 2016), la teoría de los módulos innatos (Leslie, 1992, 1999) y algunas posiciones dispersas sobre una teoría socioconstruccionista de la ToM (Liverta-Sempio, \& Marchetti, 1997), cada una con conceptos e hipótesis diversas y hasta cierto punto antagónicas, que buscan dar una explicación naturalista al problema en cuestión y más que hacer una revisión detallada de cada una se plantea que todas tienen diversos puntos de encuentro, entre ellos el hecho de que soportan la construcción teórica en la investigación empírica en el dominio de la ciencia cognitiva y de este modo encontrar los correlatos funcionales de la capacidad de un ser humano de dar cuenta de las otras mentes. También la determinación de encontrar una base material en términos evolutivos, neurales y cerebrales para explicar la capacidad de un animal humano y no humano de dar cuenta de las otras mentes.

Dada la complejidad del funcionamiento de este mecanismo en los seres humanos, se reconoce de manera fundamental el papel del medioambiente en términos de estructura social, significaciones sociales y culturales compartidos, procesos cognitivos, historia de aprendizaje del sujeto, entre otros aspectos, en los procesos funcionales neurocerebrales y el tipo de inferencia y acciones que se ejecutan.

Se ha reconocido que el problema en su versión filosófica y científica no es más que el problema de la intersubjetividad como intercambio de significados entre las personas. En este sentido, autores como Bandura y Vygotsky han aportado de manera importante en el campo de la psicología justamente porque han reclamado la importancia del vínculo intersubjetivo y el papel del aprendizaje social en el desarrollo de la cognición social. Los aportes de Piaget también se consideran relevantes porque su propuesta de una epistemología genética para entender el desarrollo evolutivo en la niñez, ubica la importante de procesos madurativos a nivel biológico en la adquisición de habilidades más complejas, entre ellas 
habilidades de orden formal como la capacidad de inferir estados mentales en los demás.

Las diversas posturas teóricas han planteado que la capacidad de dar cuenta de las otras mentes es el resultado de procesos evolutivos de la especie, en el que la capacidad de leer las intenciones de los demás tiene una función adaptativa y de supervivencia. De acuerdo con Carmona-Cañabate, (2014), en el dominio de la neurociencia cognitiva se defiende la hipótesis de que "[...] uno de los factores principales que ha guiado la evolución del hombre es la vida en sociedad y la consecuente necesidad de manejar relaciones sociales cada vez más complejas" (p. 694). De esta manera, la evolución ha requerido de procesos de agrupación social que han llevado, a su vez, a la necesidad de hacer lecturas de los deseos, creencias, intenciones y acciones de los demás como ruta adaptativa y de supervivencia. ¿Cómo formular, entonces, el problema de las otras mentes desde una visión naturalista?

El supuesto de que la capacidad de un individuo para atribuir de manera espontánea y natural estados mentales en la perspectiva de la tercera persona, se debe esencialmente al funcionamiento del cerebro y al desarrollo evolutivo que lo originó. Ha dado lugar a formas más sofisticadas de plantear el problema de las otras mentes en términos no solo epistemológicos, sino también empíricos. Desde un punto de vista científico, en el dominio de la neurociencia social, especialmente los estudios de neuronas espejo, cognición social, teoría de la mente y empatía, han presentado evidencia de que la evolución ha dotado al ser humano de estructuras y áreas cerebrales/ neurales funcionales que les permiten reconocer emociones y estados mentales en los demás hasta el punto de que la capacidad de inferir estados mentales en los demás es un elemento clave en el desarrollo de la especie y el progreso social y que de alguna manera, forma parte de un complejo proceso en el que la evolución, el aprendizaje social y la realidad institucional han permeado el desarrollo de las estructuras de la ToM. De acuerdo con lo planteado en el desarrollo de este trabajo, la forma como las neurociencias cognitivas han planteado el problema puede versar del siguiente modo:

¿Existen estructuras cerebrales y redes neurales funcionales que expliquen la capacidad de un individuo para dar cuenta de las otras mentes, en términos de sus intenciones, creencias, acciones y estados cognitivos y emocionales, de tal manera, incluso, de poder hacer inferencias que predicen acciones en los demás con base en el papel del contexto social o la realidad institucional?
Evidentemente, en coherencia con la explicación naturalista de las otras mentes, la respuesta es sí.

\section{Referencias}

Baron-Cohen, S., Leslie, A., \& Frith, U. (1985). Does the autistic children have a theory of mind? Cognition, 21, 37-46. https://psycnet.apa.org/doi/10.1016/00100277(85)90022-8

Bennett, M (2008). Neurociencia y filosofía. En M, Bennett., D, Dennett., P, Hacker y J, Searle. La naturaleza de la conciencia. Cerebro, mente y lenguaje (pp.69-93). Espańa: Paidós.

Benavides-Delgado, J., \& Roncancio-Moreno, M. (2009). Conceptos de desarrollo en estudios sobre teoría de la mente en las últimas tres décadas. Avances en Psicología Latinoamericana, 27(2), 297-310. Recuperado de https://revistas.urosario.edu.co/index.php/apl/article/viewFile/1176/1046

Brunsteins, P. (2011). El rol de la empatía en la atribución mental. Revista Argentina de Ciencias del Comportamiento, 3 (1), 75-84. Recuperado de https://revistas.unc.edu.ar/ index.php/racc/article/view/5235/5400

Carmona- Cañabate. (2014). Cognición social. En D, Redolar (comp). Neurociencia cognitiva (pp 693-716). Madrid: Medica panamericana

Churchland, P. (1995). El materialismo eliminativo y las actitudes proposicionales. En E. Rabossi (comp). Filosofía de la mente y ciencia cognitiva(pp.43-68). Barcelona: Paidós

Clark, K. J. (2016). Naturalism and its discontents. en K, L, Clark (ed.). The Blackwell Companion to Naturalism (pp. 1-15). UK: Wiley Blackwel

Damasio, A. (2011). Y el cerebro creo al hombre. Bogotá: Ed planeta Colombiana

Davidson, D. (1994). Sucesos mentales. En D, Davidson. Ensayos sobre acciones y sucesos (pp. 263-287). trad. O. Hansberg, J.A. Robles y M.M. Valdés. Barcelona: editorial Instituto de Investigaciones Filosóficas- UNAM

Dennett, D. (1998). La actitud intencional. Barcelona: Gedisa

Dennett, D. (1988b). Los verdaderos creyentes: la estrategia intencional y por qué funciona. En D. Dennett. La actitud intencional (pp. 25-49). Barcelona: Gedisa

Dennett, D. (2003). Quinear los qualia. En Obeth Hansberg y Maile Ezcurdia (comps.), La naturaleza de la experiencia. Vol. 1 Sensaciones. México, Instituto de Investigaciones Filosóficas de la UNAM.

Descartes, R. (2005[1647]). Las Meditaciones metafísicas, Madrid: Alianza.

Fodor, J. (1985). Fodor's guide to mental representations. Mind, 94, 77-110. 
Goldman, A. (2009). Mirroring, Simulating, and Mindreading. Mind \& Language, 24(2), 235-252. https://doi. org/10.1111/j.1468-0017.2008.01361.x

Goldman, A. (2006). Simulating Minds: The Philosophy, Psychology, and Neuroscience of Mindreading. Oxford: Oxford University Press

Hacker, P.M.S. (2006). Passing by the Naturalistic Turn: On Quine's Cul-de-Sac. Philosophy, 81(316), 231-253. https:// doi.org/10.1017/S003181910631604X

Iacoboni, M. (2009). The Problem of Other Minds Is Not a Problem: Mirror Neurons and Intersubjectivity. En J. A, Pineda (ed) Mirror Neuron Systems. The Role of Mirroring Processes in Social Cognition. New York: Spinger. University of California. DOI: 10.1007/978-1-59745-479-7.

Leslie, A. M. (1992). Pretense, autism and the "Theory-ofMind" module. Current Directions in Psychological Science, 1, 18-21. https://doi.org/10.1111\%2F1467-8721. ep10767818

Leslie, A. M. \& Frith, U. (1988). Autistic children's understanding of seeing, knowing and believing. British Journal of Developmental Psychology, 6(4), 315-324. http://dx.doi. org/10.1111/j.2044-835X.1988.tb01104.x

Liverta-Sempio, O. \& Marchetti, A. (1997). Cognitive developement and theories of mind: Towards a contextual approach. European Journal of psychology of Education, 12 (1), 3-21. https://doi.org/10.1007/BF03172866

Llinas, R. (2003). El cerebro y el mito del yo. El papel de las neuronas en el pensamiento y el comportamiento humanos. México: Norma, sexta reimpresión

Lewis, D. (1983). Mad pain and Martian pain. En Philosophical Papers, (pp. 122-130). Vol. I. Oxford University Press

Nagel. (2003). ¿Cómo es ser un murciélago? En Obeth Hansberg y Maile Ezcurdia (comps.), La naturaleza de la experiencia. (pp. 45-63) Vol. 1 Sensaciones. México, Instituto de Investigaciones Filosóficas de la UNAM.

Perner, J., \& Wimmer, H. (1985). Jhon thinks that Mary think's that... Atribution of second orden false belief by 5-10 years old children. Journal of Experimental Child Psychology, 39(3), 437-471. https://doi.org/10.1016/00220965(85)90051-7

Perner, J., \& Wimmer, H. (1987). Young children's understanding of belief and communicative intention. Pakistan Journal of Psychological Research, 2, 17-40. Recuperado de https:// go.gale.com/ps/anonymous?id=GALE\%7CA26163264 9\&sid=googleScholar\&v=2.1\&it=r\&linkaccess=abs\&iss $\mathrm{n}=10160604 \& \mathrm{p}=\mathrm{HRCA} \& \mathrm{sw}=\mathrm{w}$

Pineda, J. A., A, Moore, R., Elfenbeinand, H., \& Cox, R. (2009). Hierarchically Organized Mirroring Processes in Social Cognition: The Functional Neuroanatomy of Empathy. En J. A, Pineda (ed.) Mirror Neuron Systems. The Role of Mirroring Processes in Social Cognition. New York:
Spinger. University of California. DOI: 10.1007/978-159745-479-7.

Premack D, Woodruff G. (1978). Does chimpanzee have a theory of mind? Behavioral and Brain Sciences, 1 (4), 515526. https://doi.org/10.1017/S0140525X00076512

Quine, W. V. (1960). Word and Object. Cambirdge, Mass, The MIT Press

Quine, W.V.O. (1984 [1969]). Epistemología Naturalizada. En W.V.O. Quine. La Relatividad ontológica y otros ensayos, Madrid: Tecnos.

Quine, W.V.O. (1975). Mind and verbal dispositions. En S. Guttenplan (ed). Mind and lenguaje (pp.83-95). Oxford: Clarendon press,

Quine, W, V. (1998). Cosas de la mente. En W, V, Quine. Del estímulo a la ciencia. Barcelona: Ariel,

Scotto, C. (2017). Del problema de las “otras mentes” a la cognición social: una defensa de la epistemología naturalizada. Epistemología e Historia de la Ciencia, 2(1), 43-66.

Searle, J.R. (1996). El redescubrimiento de la mente. Barcelona: Crítica.

Searle, J. R. (1985). Mentes, cerebros y ciencia. Madrid: Ediciones Cátedra

Searle, J. R (2006). La mente. Una breve introducción. Colombia: Ed. Norma.

Stich, S. (1983). From Folk Psychology to Cognitive Science, Cambridge, MA: MIT Press

Tononi, G \& Koch, C. (2008). The neural correlates of consciousness: an update. Annals of the New York Academy of Sciences, 1124(1):239-261. https://doi.org/10.1196/ annals. 1440.004

Wellman, H. M \& Liu, D. (2004). Scaling of theory-ofmind tasks. Child Development, 75, 523-541. https://doi. org/10.1111/j.1467-8624.2004.00691.x

Wellman, H. M. (2014). Making Minds, How Theory of Mind Develops. Oxford Series in Cognitive Development, Oxford: Oxford University Press.

Wellman, H. M. (1990). The Child's Theory of the Mind. Cambridge: MIT Press.

Wittgenstein, L. (1988 [1953]). Investigaciones filosóficas. México: UNAM, 243-315

Wittgenstein, L. (1996). Últimos escritos sobre filosofía de la psicología. Madrid: Ed Tecnos

Wimmer, H., \& Perner, J. (1983). Beliefs about beliefs: representation and constraining functions of wrong beliefs in young children's understanding of deception. Cognition, 13 (1), 103-128. https://psycnet.apa.org/doi/10.1016/00100277(83)90004-5

$70<$ Universidad de San Buenaventura, Cali, Colombia 